\title{
Dysplastic naevi with moderate to severe histological dysplasia: a risk factor for melanoma
}

\author{
A.R. Shors, S. Kim, E. White, ${ }^{\star}$ Z. Argenyi, R.L. Barnhill, $\uparrow$ P. Duray, $\$$ L. Erickson,§ J. Guitart,

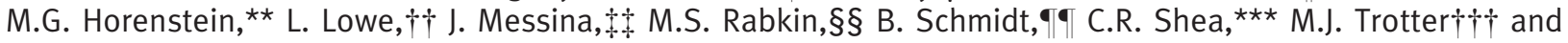 \\ M.W. Piepkorn \\ Department of Medicine/Dermatology, University of Washington Medical Center, Seattle, WA, U.S.A. \\ *Cancer Prevention, Fred Hutchinson Cancer Research Center, Seattle, WA, U.S.A. \\ $\dagger$ Departments of Dermatology and Pathology, University of Miami, Miami, FL, U.S.A. \\ †Department of Pathology, West Roxbury Veterans Affairs Medical Center, West Roxbury, MA, U.S.A. \\ $\S$ Department of Pathology and Laboratory Medicine, Mayo Clinic, Rochester, MN, U.S.A. \\ -Department of Dermatology, Northwestern University, Chicago, IL, U.S.A. \\ **Dermatopathology, The Dermatology Group, Verona, NJ, U.S.A. \\ ††Department of Pathology, University of Michigan, Ann Arbor, MI, U.S.A. \\ ttDepartment of Pathology and Medicine, University of South Florida College of Medicine, Tampa, FL, U.S.A. \\ $\S \S$ Rabkin Dermatopathology Laboratory, P.C., Pittsburgh, PA, U.S.A. \\ -Departments of Dermatology and Pathology, Children’s Hospital, Harvard University Medical Center, Boston, MA, U.S.A. \\ ***Department of Medicine, University of Chicago, Chicago, IL, U.S.A. \\ $\dagger+\dagger$ Calgary Laboratory Services, Department of Pathology and Laboratory Medicine, University of Calgary, Calgary, AB, Canada
}

\section{Summary}

\section{Correspondence}

A.R. Shors.

E-mail: ashors@u.washington.edu;

shors.a@ghc.org

\section{Accepted for publication}

12 May 2006

\section{Key words}

atypical naevi, case-control, dysplastic naevi, melanoma

\section{Conflicts of interest}

None declared.
Background The risk of malignant melanoma associated with histologically dysplastic naevi (HDN) has not been defined. While clinically atypical naevi appear to confer an independent risk of melanoma, no study has evaluated the extent to which HDN are predictive of melanoma.

Objectives To estimate the risk of melanoma associated with HDN. Secondarily, the risk associated with number of naevi and large naevi is estimated.

Methods We enrolled 80 patients with newly diagnosed melanoma along with 80 spousal controls. After obtaining information on melanoma risk factors and performing a complete cutaneous examination, the most clinically atypical naevus was biopsied in both cases and controls. Histological dysplasia was then assessed independently by 13 dermatopathologists ( 0 , no dysplasia; 1 , mild dysplasia; 2 , moderate dysplasia; 3, severe dysplasia). The dermatopathologists were blinded as to whether the naevi were from melanoma subjects or controls. Multivariate analyses were performed to determine if there was an independent association between the degree of histological dysplasia in naevi and a personal history of melanoma.

Results In persons with naevi receiving an average score of $>1$ (i.e. naevi considered to have greater than mild histological dysplasia), there was an increased risk of melanoma [odds ratio (OR) $2 \cdot 60$, 95\% confidence interval (CI) 0.99-6.86] which persisted after adjustment for confounders (OR 3.99, 95\% CI 1.0215.71). Very few dermatopathologists reliably graded naevi of subjects with melanoma as being more dysplastic than naevi of control subjects. Among the entire group, the interobserver reliability associated with grading histological dysplasia in naevi was poor (weighted kappa $0 \cdot 28$ ).

Conclusions HDN do appear to confer an independent risk of melanoma. However, this result may add more to our biological understanding of melanoma risk than to clinical assessment of risk, because HDN assessed by a single pathologist generally cannot be used to assess risk of melanoma. Future studies should be directed at establishing reproducible, predictive criteria for grading naevi. 
The atypical or dysplastic melanocytic naevus accounts for 650000 new patient visits annually to U.S. dermatologists. ${ }^{1}$ It is a defining feature of melanoma high-risk groups such as the atypical mole syndrome, although it occurs much more commonly as a sporadic finding in otherwise healthy individuals. Despite their prevalence, sporadic dysplastic naevi remain a poorly defined entity. A recent survey of dermatologists suggests that there exists a substantial degree of variation in how the dysplastic naevus is defined and treated. ${ }^{1}$ Others remain sceptical of its existence. ${ }^{2}$ When defined clinically (pigmented lesions $>5 \mathrm{~mm}$ with any number of features including colour variegation, border irregularity and asymmetry) atypical naevi appear to confer an independent risk for malignant melanoma. $^{3-8}$ However, the histopathological correlates of clinical atypia are inconsistent, ${ }^{9}$ and while some advocate defining the dysplastic naevus histologically, ${ }^{10,11}$ no study has determined melanoma risk associated with histological dysplasia. Previous studies have found melanocytic dysplasia to be a common histological finding in the general white-skinned population, suggesting that it is not a strong predictor of melanoma. ${ }^{12}$

Given this controversy, we utilized a case-control design to evaluate the risk of invasive malignant melanoma associated with histologically dysplastic naevi (HDN). We further examined whether this relationship was independent of the effects of naevus count or the presence of large naevi, which are risk factors for melanoma $a^{4,5,7,13}$ and correlate with dysplastic naevi. ${ }^{14}$

\section{Patients and methods}

\section{Subject selection}

Eighty cases of invasive malignant melanoma were enrolled between 1998 and 2001. Cases were patients with incident melanoma under evaluation in outpatient dermatology and oncology clinics at the University of Washington Medical Center. In addition, 80 unaffected spousal controls were accrued. In two cases, same-sex domestic partners were enrolled as controls. A matched design was chosen to minimize variation in other risk factors (thus increasing precision of the model), as spouses tend to be matched on a number of important melanoma risk factors such as race, residence and socioeconomic status. Spouses were also selected as the control group because they are typically motivated to participate in medical studies, and we asked controls to undergo a cutaneous examination and biopsy. The study was approved by the Institutional Review Board of the Human Subjects Division, University of Washington Medical Center.

\section{Data collection}

Full cutaneous examinations were performed by a dermatology fellow (S.K.). The most clinically atypical macular naevus was biopsied in both cases and controls. Clinical atypia was subjectively assessed by a combination of size, macularity, colour variegation, asymmetry and border irregularity. Thirteen dermatopathologists separately reviewed all histological sections blinded to the status of the subject, and each assigned a dysplasia score of: 0 , no dysplasia; 1 , mild dysplasia; 2, moderate dysplasia; or 3, severe dysplasia. All dermatopathologists have a special interest in melanocytic lesions and interpret atypical (dysplastic) naevi on a daily basis. In order to re-create the current standard of practice, there was no discussion or consensus about criteria in grading histological dysplasia prior to the review of the study set. All the dermatopathologists graded dysplasia by the criteria they use in their daily practice, based on their understanding of the medical literature. The basic features of naevomelanocytic dysplasia have been published elsewhere. ${ }^{10,15,16}$

Naevus counts were performed on the back. An individual was considered to have large naevi if a single naevus on the back measured $>5 \mathrm{~mm}$. Freckles on the shoulders (0-10, $11-50,>50)$ were likewise tabulated, in addition to eye colour and Fitzpatrick skin type (I-VI). Hair colour was obtained by asking the participant their natural hair colour at 15 years. Enrolled subjects completed a questionnaire concerning demographic characteristics including age, education, income, current residence, birth residence and occupation. In addition, information on personal cancer history (all-site cancer history and specifically nonmelanoma and melanoma skin cancers) and family history of melanoma in first-degree relatives was obtained. Sunburn history was defined as the lifetime number of blistering sunburns. Sensitivity to intermittent and chronic sun exposure was estimated by asking participants their tendency to burn ('If your skin is exposed to strong sunlight for the first time in summer for $1 \mathrm{~h}$, would you: get a severe sunburn with blistering; have a painful sunburn for a few days followed by peeling; get mildly burnt followed by some degree of tanning; tan without any sunburn?') and ability to tan ('After repeated and prolonged exposure to sunlight your skin becomes: deeply tanned; moderately tanned; only mildly tanned; only freckled or no suntan at all?'). Other estimates of ultraviolet (UV) exposure were obtained, including the number of weeks spent in sunny resorts, use of tanning beds or use of UV radiation for medical conditions.

\section{Data analysis}

The three main risk factors of interest were the dysplasia score of the most clinically atypical naevus (obtained as a group average of each dermatopathologist's score), number of naevi on the back and presence or absence of a large naevus. Risk of melanoma associated with dysplasia was calculated for individual pathologists as well as for the overall group-averaged dysplasia score. The average score is reported as a dichotomous variable $(\leq 1$ and $>1)$. Several cutoffs were considered. The strongest risk of melanoma was seen for naevi with an average dysplasia score of $>1$. Because this group can also be interpreted as naevi with greater than mild dysplasia, it was chosen as the cutoff. A weighted kappa statistic was calculated to estimate the interobserver reliability between pathologists in grading mild, moderate, severe and no dysplasia. A weighted 
kappa statistic accounts for the magnitude of disagreement when using ordinal data. ${ }^{17}$

Given the inherent matching with a spousal referent group, conditional logistic regression was performed in all analyses. Odds ratios (ORs) and 95\% confidence intervals (CIs) for the relation between the risk factors and melanoma risk were computed as age- and sex-adjusted ORs, as well as a fully adjusted OR, which included adjustment of all confounders. Inclusion of potential confounders in the fully adjusted model was determined by addition of the strongest predictors in a stepwise fashion into the logistic regression model. To establish the relative contribution of large naevi, naevus counts and histological dysplasia to melanoma risk, each of these variables was also reported with mutual adjustment of the other predictors. Data analysis was performed using Intercooled STATA 8.0 software (College Station, TX, U.S.A.).

\section{Results}

Table 1 presents the distribution of demographic and melanoma risk factors in the study and gives the estimated risk of melanoma associated with these factors in this population. Cases were $56 \%$ male, with a mean age of 47 years (range 25-75), and controls were $41 \%$ male with a mean age of 48 years (range $23-77$ ). Given that spouses are to some extent matched on age, CIs for the risk of melanoma with age were wide and nonsignificant, although cases tended to be older than controls. Both groups were generally college educated. More cases than controls attended college or obtained a graduate degree, and while this was not statistically significant, variance estimates were wide, consistent with some degree of matching. History of nonmelanoma skin cancer was strongly associated with melanoma risk, as to a lesser extent was family history of melanoma. Personal characteristics such as red or blond hair, blue or green eyes, and Fitzpatrick skin type I and II were all associated with melanoma risk. More than 50 freckles on the shoulder was a significant risk factor for melanoma (OR 3.72, 95\% CI 1·23-11·24). A lifetime history of more than six blistering sunburns was also statistically significant (OR 4.58, 95\% CI 1·23-17·00). More cases than controls had a strong tendency to burn and no or mild ability to tan.

Table 2 presents the estimated risk of melanoma associated with histological dysplasia in naevi, presence of a large naevus (> $5 \mathrm{~mm}$ ) on the back, and naevus counts on the back. ORs and 95\% CIs were calculated after adjustment for age and sex, as well as in a full model that included the dysplasia score, naevus counts on the back and large naevi, as well as eye colour, Fitzpatrick skin type, history of severe sunburns and family history of melanoma. The presence of at least one large naevus on clinical examination was not significantly associated with melanoma risk (age- and sex-adjusted OR 1.81, 95\% CI 0·75-4·37, fully adjusted model OR $2 \cdot 52$, 95\% CI 0·67-9·42). Naevus counts were associated with melanoma risk (for $\geq 10$ naevi on back: age- and sex-adjusted OR 3.68, 95\% CI $1 \cdot 38-$ 9.80, $\mathrm{P}$ for trend $0 \cdot 01$, fully adjusted model OR $4 \cdot 25,95 \% \mathrm{CI}$ 0.99-18.3, P for trend 0.04). Average dysplasia scores were
Table 1 Age- and sex-adjusted odds ratios (ORs) and 95\% confidence intervals (CIs) for risk of melanoma

\begin{tabular}{|c|c|c|c|}
\hline Risk factor & Cases & Controls & OR $(95 \% \mathrm{CI})$ \\
\hline \multicolumn{4}{|l|}{ Sex } \\
\hline Female & 35 & 47 & 1 \\
\hline Male & 45 & 33 & $1 \cdot 25(0.78-2 \cdot 02)$ \\
\hline \multicolumn{4}{|l|}{ Age (years) } \\
\hline$<41$ & 27 & 28 & 1 \\
\hline $41-51$ & 26 & 29 & $1 \cdot 47(0 \cdot 12-18 \cdot 03)$ \\
\hline$>51$ & 27 & 23 & $3 \cdot 37(0 \cdot 21-55 \cdot 12)$ \\
\hline \multicolumn{4}{|l|}{ Education } \\
\hline High school & 13 & 17 & 1 \\
\hline College & 51 & 49 & $2 \cdot 23(0.75-6 \cdot 64)$ \\
\hline Graduate degree & 15 & 14 & $2.56(0.61-10.83)$ \\
\hline \multicolumn{4}{|l|}{ History of NMSC } \\
\hline No & 65 & 77 & 1 \\
\hline Yes & 14 & 3 & $11.89(1.52-92.53)$ \\
\hline \multicolumn{4}{|c|}{ Family history of melanoma } \\
\hline No & 61 & 73 & 1 \\
\hline Yes & 17 & 7 & $2 \cdot 91(1 \cdot 05-8 \cdot 10)$ \\
\hline \multicolumn{4}{|l|}{ Hair colour } \\
\hline Brown/black & 49 & 63 & 1 \\
\hline Red/blond & 31 & 17 & $2 \cdot 72(1.23-6.03)$ \\
\hline \multicolumn{4}{|l|}{ Eye colour } \\
\hline Brown & 8 & 24 & 1 \\
\hline Blue & 42 & 37 & $5 \cdot 4(1.55-18 \cdot 83)$ \\
\hline Green & 30 & 19 & $9 \cdot 08(2 \cdot 27-36 \cdot 29)$ \\
\hline \multicolumn{4}{|l|}{ Fitzpatrick skin type } \\
\hline III/IV & 48 & 60 & 1 \\
\hline I/II & 32 & 20 & $2 \cdot 25(1 \cdot 10-4 \cdot 60)$ \\
\hline \multicolumn{4}{|l|}{ Freckles, shoulders } \\
\hline $0-10$ & 17 & 31 & 1 \\
\hline $11-50$ & 22 & 18 & $2 \cdot 62(0.94-7 \cdot 31)$ \\
\hline$>50$ & 41 & 31 & $3 \cdot 72(1 \cdot 23-11 \cdot 24)$ \\
\hline \multicolumn{4}{|c|}{ Blistering burns (lifetime) } \\
\hline None & 10 & 18 & 1 \\
\hline $1-3$ & 37 & 39 & $2 \cdot 12(0.67-6.63)$ \\
\hline $4-6$ & 12 & 12 & $2.54(0.61-10.54)$ \\
\hline$>6$ & 20 & 11 & $4 \cdot 58(1 \cdot 23-17 \cdot 00)$ \\
\hline \multicolumn{4}{|l|}{ Tendency to burn } \\
\hline Mild or no burn & 43 & 49 & 1 \\
\hline $\begin{array}{l}\text { Severe or painful } \\
\text { burn }\end{array}$ & 36 & 31 & $1 \cdot 37(0 \cdot 70-2 \cdot 68)$ \\
\hline \multicolumn{4}{|l|}{ Ability to $\tan$} \\
\hline Moderate to deep & 50 & 56 & 1 \\
\hline None or mild & 29 & 20 & $2 \cdot 16(0.99-4.72)$ \\
\hline
\end{tabular}

NMSC, nonmelanoma skin cancer.

low, with only $17 \cdot 5 \%$ of specimens receiving an average score $>1$, and only one biopsy receiving an average score $>2$. An average dysplasia score of $>1$ was associated with a statistically significantly increased risk of melanoma in the age- and sex-adjusted model (OR 2.60, 95\% CI 0.99-6.86), and remained an independent risk factor after adjustment for number of naevi and other risk factors (OR 3.99, 95\% CI 1.0215.71).

Table 3 presents each individual pathologist's dysplasia score. This reflects to what degree melanoma subjects' naevi 
Table 2 Odds ratios (ORs) and 95\% confidence intervals (CIs) reporting the relative contribution of histological dysplasia in naevi, ${ }^{a}$ naevus counts and presence of at least one large naevus ( $>5 \mathrm{~mm}$ ) towards melanoma risk

\begin{tabular}{|c|c|c|c|c|}
\hline Risk factor & Cases & Controls & OR $(95 \% \mathrm{CI})^{\mathrm{b}}$ & OR $(95 \% \mathrm{CI})^{\mathrm{c}}$ \\
\hline \multicolumn{5}{|c|}{ Average dysplasia score } \\
\hline$\leq 1$ & 61 & 71 & 1 & 1 \\
\hline$>1$ & 19 & 9 & $2.60(0.99-6.86)$ & $3.99(1.02-15.71)$ \\
\hline \multicolumn{5}{|l|}{ Naevi on back } \\
\hline $0-4$ & 12 & 23 & 1 & 1 \\
\hline $5-9$ & 17 & 28 & $1 \cdot 27(0 \cdot 48-3 \cdot 34)$ & $0 \cdot 71(0 \cdot 19-2 \cdot 69)$ \\
\hline$\geq 10$ & 51 & 29 & $3 \cdot 68(1 \cdot 38-9 \cdot 80)$ & $4 \cdot 25(0 \cdot 99-18 \cdot 3)$ \\
\hline P for trend & & & 0.01 & $0 \cdot 04$ \\
\hline \multicolumn{5}{|l|}{ Naevi $>5 \mathrm{~mm}$} \\
\hline None on back & 57 & 65 & 1 & 1 \\
\hline At least 1 on back & 23 & 15 & $1 \cdot 81(0 \cdot 75-4 \cdot 37)$ & $2 \cdot 52(0.67-9 \cdot 42)$ \\
\hline
\end{tabular}

demonstrated more histological dysplasia than naevi from control subjects, as graded by individual pathologists blinded to the status of cases and controls. Of the 13 pathologists, only one had scores which significantly correlated with melanoma risk. This individual graded naevi more often as moderate or severe in melanoma subjects (OR 5.52, 95\% CI 1·13-26.97), although the scores of several others had high but not statistically significant ORs. Interobserver reliability was poor, with a weighted kappa statistic of $0 \cdot 28$. However, when a group average was used, the histological grading was predictive of melanoma.

\section{Discussion}

We found HDN to be more common in patients with melanoma than in controls. This association was independent of naevus counts, as well as other well-known risk factors for melanoma. Our utilization of 13 pathologists provides stability to an entity which is variably defined and recognized. While there are numerous case-control studies examining the importance of clinically atypical naevi, this is the first study designed to evaluate an independent melanoma risk associated with HDN.

Several previous studies have suggested an association between HDN and melanoma. In a retrospective review of HDN, persons with a history of melanoma were more likely to have a severely (OR $4 \cdot 08,95 \%$ CI $2 \cdot 91-5 \cdot 7$ ) or moderately (OR $1 \cdot 45$, 95\% CI 1.13-1.87) dysplastic naevus vs. a mildly dysplastic naevus. This study depended on providers reporting a melanoma history in the pathology accession, and adjustment for other confounders such as naevus counts could not be performed. ${ }^{18}$ A similar study of archived pathology data found that $57 \%$ of persons with HDN had a history of melanoma, while if the naevus was considered 'questionably dysplastic' or nondysplastic, only $25 \%$ and $18 \%$ had a history of melanoma, respectively. ${ }^{19}$ Bergman et al. ${ }^{20}$ similarly reported that markedly atypical naevi were more likely to arise in persons with a history of melanoma. Half of their cohort belonged to melanoma kindreds, a small subpopulation clearly at increased risk of melanoma, making the results difficult to interpret.

Other studies have not demonstrated a relationship between melanoma risk and histological dysplasia. Piepkorn et al. ${ }^{12}$ found mild dysplasia in the naevi of $7-32 \%$ of a healthy population, suggesting that dysplasia is common and not a strong predictor of melanoma. Another study compared naevi from persons with dysplastic naevi with a control population, finding a nonsignificant trend towards more dysplasia among the group with dysplastic naevi. No adjustments for confounders could be performed. ${ }^{15}$ Klein and Barr ${ }^{21}$ examined clinically benign naevi in healthy individuals, and found that $88 \%$ had at least one feature of dysplasia, and $29 \%$ had three features. Our study does demonstrate an independent risk associated with HDN, although the interpretation of our cutoff is important. All naevi in the study (both cases and controls) were graded as at least mildly dysplastic by at least one pathologist. The excess risk was seen only in naevi in which the group average was $>1$, or naevi in which the average group score was greater than mild. Most naevi graded as mild by an individual pathologist are not associated with an increased risk of melanoma. These results are also corroborated by a previous study showing that one begins to observe DNA aneuploidy in dysplastic naevi that are graded as moderately atypical. ${ }^{22}$

HDN appear to be an independent risk factor for melanoma, perhaps reflecting the pleiotropic effects of genetic and environmental aetiological factors that are common between the two entities. A population-based study evaluated biopsies of the two most clinically atypical naevi from a single individual, and demonstrated a significant correlation, suggesting that some persons have a predisposition to melanocytic dysplasia. ${ }^{23}$ Some argue that a substantial proportion of melanomas arises from dysplastic naevi. Our study was not designed to examine any potential precursor role of the HDN. To date, no study has evaluated the prophylactic excision of dysplastic naevi as a modality of melanoma prevention. 


\begin{tabular}{|c|c|c|c|}
\hline Pathologist & Cases & Controls & OR $(95 \% \mathrm{CI})^{\mathrm{a}}$ \\
\hline \multicolumn{4}{|l|}{1} \\
\hline No dysplasia & 34 & 37 & 1 \\
\hline Mild dysplasia & 34 & 27 & $1 \cdot 35(0 \cdot 64-2 \cdot 82)$ \\
\hline Moderate or severe dysplasia & 12 & 16 & $0.64(0.24-1 \cdot 65)$ \\
\hline \multicolumn{4}{|l|}{2} \\
\hline No dysplasia & 29 & 29 & 1 \\
\hline Mild dysplasia & 40 & 44 & $0.75(0.35-1.59)$ \\
\hline Moderate or severe dysplasia & 11 & 7 & $1 \cdot 32(0 \cdot 41-4 \cdot 18)$ \\
\hline \multicolumn{4}{|l|}{3} \\
\hline No dysplasia & 37 & 36 & 1 \\
\hline Mild dysplasia & 20 & 26 & $0.67(0.29-1.59)$ \\
\hline Moderate or severe dysplasia & 23 & 18 & $1.07(0.47-2 \cdot 47)$ \\
\hline \multicolumn{4}{|l|}{4} \\
\hline No dysplasia & 48 & 51 & 1 \\
\hline Mild dysplasia & 26 & 26 & $1.00(0.49-1.99)$ \\
\hline Moderate or severe dysplasia & 6 & 3 & $2 \cdot 01(0 \cdot 44-9 \cdot 29)$ \\
\hline \multicolumn{4}{|l|}{5} \\
\hline No dysplasia & 58 & 66 & 1 \\
\hline Mild dysplasia & 19 & 12 & $1.61(0.72-3 \cdot 61)$ \\
\hline Moderate or severe dysplasia & 3 & 2 & $1.50(0.24-9.51)$ \\
\hline \multicolumn{4}{|l|}{6} \\
\hline No dysplasia & 49 & 53 & 1 \\
\hline Mild dysplasia & 22 & 25 & $0.86(0.41-1.81)$ \\
\hline Moderate or severe dysplasia & 9 & 2 & $7 \cdot 05(0.85-58.33)$ \\
\hline \multicolumn{4}{|l|}{7} \\
\hline No dysplasia & 44 & 44 & 1 \\
\hline Mild dysplasia & 29 & 35 & $0 \cdot 84(0 \cdot 44-1 \cdot 61)$ \\
\hline Moderate or severe dysplasia & 7 & 1 & $6 \cdot 20(0 \cdot 72-52 \cdot 63)$ \\
\hline \multicolumn{4}{|l|}{8} \\
\hline No dysplasia & 37 & 46 & 1 \\
\hline Mild dysplasia & 25 & 19 & $1.52(0 \cdot 72-3 \cdot 22)$ \\
\hline Moderate or severe dysplasia & 18 & 15 & $1 \cdot 30(0 \cdot 54-3 \cdot 12)$ \\
\hline \multicolumn{4}{|l|}{9} \\
\hline No dysplasia & 40 & 52 & 1 \\
\hline Mild dysplasia & 30 & 24 & $1 \cdot 65(0 \cdot 80-3 \cdot 44)$ \\
\hline Moderate or severe dysplasia & 10 & 4 & $5 \cdot 02(0.96-26 \cdot 39)$ \\
\hline \multicolumn{4}{|l|}{10} \\
\hline No dysplasia & 27 & 32 & 1 \\
\hline Mild dysplasia & 32 & 35 & $0.98(0.49-1.98)$ \\
\hline Moderate or severe dysplasia & 21 & 13 & $2 \cdot 00(0 \cdot 76-5 \cdot 23)$ \\
\hline \multicolumn{4}{|l|}{11} \\
\hline No dysplasia & 37 & 37 & 1 \\
\hline Mild dysplasia & 24 & 31 & $0.65(0.30-1.39)$ \\
\hline Moderate or severe dysplasia & 19 & 12 & $1.50(0.53-4.25)$ \\
\hline \multicolumn{4}{|l|}{12} \\
\hline No dysplasia & 44 & 49 & 1 \\
\hline Mild dysplasia & 23 & 26 & $0.85(0.43-1 \cdot 70)$ \\
\hline Moderate or severe dysplasia & 13 & 5 & $5 \cdot 52(1.13-26.97)$ \\
\hline \multicolumn{4}{|l|}{13} \\
\hline No dysplasia & 67 & 72 & 1 \\
\hline Mild dysplasia & 12 & 8 & $2 \cdot 13(0.75-6.01)$ \\
\hline Moderate or severe dysplasia & 1 & 0 & $2 \cdot 3 \mathrm{E}+14(0-\infty)$ \\
\hline
\end{tabular}

Table 3 Risk of melanoma for individual pathologists' interpretation of dysplasia
Several limitations should be mentioned. The potential for recall bias exists for many of our covariates such as personal or family history of cancer, number of sunburns and sun sen- sitivity. Assessment of pigmentary characteristics by a single clinical dermatologist unblinded to the status of subjects is a possible source of bias. In addition, having a single 
dermatologist choose the most clinically atypical naevi may not be generalizable. However, it is difficult to imagine how this might have systematically contributed to a bias. Importantly, no biopsied specimen was considered to be an entity other than a melanocytic naevus by the majority of pathologists. If melanomas removed in the cases arose from HDN, our estimates of risk associated with HDN may be conservative. Lastly, the cumulative dysplasia scores were low, which accounts for the variance in our estimates of the risk associated with moderate to severe dysplasia.

The variability in grading of dysplasia is troubling. Our kappa statistic of $0 \cdot 28$ reflects agreement on a group of naevi which were on average mildly dysplastic and cannot be compared directly with previous studies that have examined samples more representative of the spectrum of atypia. None the less, most other studies demonstrate similar poor to fair interobserver reliability. ${ }^{12,24}$ When predefined criteria are used, interobserver agreement generally improves somewhat. ${ }^{25-27}$ Future studies should determine which histological features are most useful in predicting melanoma risk so that universal criteria can be developed.

\section{References}

1 Tripp JM, Kopf AW, Marghoob AA, Bart RS. Management of dysplastic nevi: a survey of fellows of the American Academy of Dermatology. J Am Acad Dermatol 2002; 46:674-82.

2 Ackerman AB. What naevus is dysplastic, a syndrome and the commonest precursor of malignant melanoma? A riddle and an answer. Histopathology 1988; 13:241-56.

3 Roush GC, Nordlund JJ, Forget B et al. Independence of dysplastic nevi from total nevi in determining risk for nonfamilial melanoma. Prev Med 1988; 17:273-9.

4 Tucker MA, Halpern A, Holly EA et al. Clinically recognized dysplastic nevi. A central risk factor for cutaneous melanoma. JAMA 1997; 277:1439-44.

5 Holly EA, Kelly JW, Shpall SN, Chiu SH. Number of melanocytic nevi as a major risk factor for malignant melanoma. J Am Acad Dermatol 1987; 17:459-68.

6 Halpern AC, Guerry D IV, Elder DE et al. Dysplastic nevi as risk markers of sporadic (nonfamilial) melanoma. A case-control study. Arch Dermatol 1991; 127:995-9.

7 Garbe C, Buttner P, Weiss J et al. Risk factors for developing cutaneous melanoma and criteria for identifying persons at risk: multicenter case-control study of the Central Malignant Melanoma Registry of the German Dermatological Society. J Invest Dermatol 1994; 102:695-9.

8 Bataille V, Bishop JA, Sasieni P et al. Risk of cutaneous melanoma in relation to the numbers, types and sites of naevi: a case-control study. Br J Cancer 1996; 73:1605-11.

9 Annessi G, Cattaruzza MS, Abeni D et al. Correlation between clinical atypia and histologic dysplasia in acquired melanocytic nevi. J Am Acad Dermatol 2001; 45:77-85.
$10 \mathrm{NIH}$ Consensus conference. Diagnosis and treatment of early melanoma. JAMA 1992; 268:1314-19.

11 Elder DE, Green MH, Guerry D IV et al. The dysplastic nevus syndrome: our definition. Am J Dermatopathol 1982; 4:455-60.

12 Piepkorn MW, Barnhill RL, Cannon-Albright LA et al. A multiobserver, population-based analysis of histologic dysplasia in melanocytic nevi. J Am Acad Dermatol 1994; 30:707-14.

13 Grob JJ, Gouvernet J, Aymar D et al. Count of benign melanocytic nevi as a major indicator of risk for nonfamilial nodular and superficial spreading melanoma. Cancer 1990; 66:387-95.

14 Roush GC, Barnhill R. Correlation of clinical pigmentary characteristics with histopathologically-confirmed dysplastic nevi in nonfamilial melanoma patients. Studies of melanocytic nevi IX. Br J Cancer 1991; 64:943-7.

15 Ahmed I, Piepkorn MW, Rabkin MS et al. Histopathologic characteristics of dysplastic nevi. Limited association of conventional histologic criteria with melanoma risk group. J Am Acad Dermatol 1990; 22:727-33.

16 Barnhill RL. Malignant melanoma, dysplastic melanocytic nevi, and Spitz tumors. Histologic classification and characteristics. Clin Plast Surg 2000; 27:331-60, viii.

17 Cohen J. Weighted kappa: nominal scale agreement with provision for scaled disagreement or partial credit. Psychol Bull 1968; 70:213-20.

18 Arumi-Uria M, McNutt NS, Finnerty B. Grading of atypia in nevi: correlation with melanoma risk. Mod Pathol 2003; 16:764-71.

19 Black WC, Hunt WC. Histologic correlations with the clinical diagnosis of dysplastic nevus. Am J Surg Pathol 1990; 14:44-52.

20 Bergman W, Ruiter DJ, Scheffer E, van Volten WA. Melanocytic atypia in dysplastic nevi. Immunohistochemical and cytophotometrical analysis. Cancer 1988; 61:1660-6.

21 Klein LJ, Barr RJ. Histologic atypia in clinically benign nevi. A prospective study. J Am Acad Dermatol 1990; 22:275-82.

22 Schmidt B, Weinberg DS, Hollister K, Barnhill RL. Analysis of melanocytic lesions by DNA image cytometry. Cancer 1994; 73:29717.

23 Piepkorn M, Meyer LJ, Goldgar D et al. The dysplastic melanocytic nevus: a prevalent lesion that correlates poorly with clinical phenotype. J Am Acad Dermatol 1989; 20:407-15.

24 Duncan LM, Berwick M, Bruijn JA et al. Histopathologic recognition and grading of dysplastic melanocytic nevi: an interobserver agreement study. J Invest Dermatol 1993; 100:S318-21.

25 Clemente C, Cochran AJ, Elder DE et al. Histopathologic diagnosis of dysplastic nevi: concordance among pathologists convened by the World Health Organization Melanoma Programme. Hum Pathol 1991; 22:313-19.

26 de Wit PE, van't Hof-Grootenboer B, Ruiter DJ et al. Validity of the histopathological criteria used for diagnosing dysplastic naevi. An interobserver study by the pathology subgroup of the EORTC Malignant Melanoma Cooperative Group. Eur J Cancer 1993; 29A:831-9.

27 Weinstock MA, Barnhill RL, Rhodes AR, Brodsky GL. Reliability of the histopathologic diagnosis of melanocytic dysplasia. The Dysplastic Nevus Panel. Arch Dermatol 1997; 133:953-8. 